\title{
REPRESENTACIONES SOCIALES DE LOS ESTUDIANTES UNIVERSITARIOS DE GRADO SOBRE LA ESCRITURA ACADÉMICA
}

\author{
SOCIAL REPRESENTATIONS AMONG GRADUATE STUDENTS ON ACADEMIC WRITING
}

REPRÉSENTATIONS SOCIALES D'ÉTUDIANTS UNIVERSITAIRES SUR L'ÉCRITURE ACADÉMIQUE

\author{
Alba Naroa Romero González \\ Doctora en Educación, máster de \\ Formación del Profesorado de \\ Secundaria, Bachiller, Formación \\ Profesional y Escuelas de Idiomas, \\ Universidad de Deusto, Bilbao, \\ España. Profesora de Educación \\ Secundaria y Bachillerato en la Ins- \\ titución de Educación Superior (IES) \\ Ibarrekolanda. \\ Universidad de Deusto, Avenida \\ de las Universidades, 24.48007 \\ (Bilbao). \\ alromer@opendeusto.es \\ ORCID: 0000-0003-2855-1130
}

\section{Manuela Álvarez Álvarez}

Doctora en Filología Hispánica por la Universidad de Deusto. Profesora titular y miembro del equipo de investigación eDucaR, Departamento de Lenguas Modernas y Estudios Vascos, Facultad de Ciencias Sociales y Humanas de la Universidad de Deusto, Bilbao, España.

Universidad de Deusto, Avenida

de las Universidades, 24.48007

(Bilbao).

manuela.alvarez@deusto.es

ORCID: 0000-0003-1187-4857

Título de la investigación: "La escritura académica de estudiantes de grado a través de sus producciones: la reseña de formación. Estudio de caso de los estudiantes de Lenguas Modernas". Tesis

\section{RESUMEN}

El conocimiento de lo que piensan los estudiantes sobre la escritura académica resulta ser una fuente de información valiosa que puede tener repercusiones importantes en propuestas didácticas más ajustadas a las necesidades formativas. Los objetivos de este estudio son indagar sobre la concepción que de escritura académica tienen los estudiantes; sobre si reciben formación respecto a este tema y qué formación es esa; y sobre la percepción de sí mismos como escritores desde que ingresaron en la universidad. La población está constituida por los 96 estudiantes del grado en Lenguas Modernas de la Universidad de Deusto, quienes han producido unas narrativas a efectos de este estudio transversal de corte cualitativo. Los resultados sugieren que la inmersión en una nueva comunidad discursiva les plantea nuevos retos para los que reciben una formación que consideran insuficiente e inadecuada, y aunque perciben una mejoría como escritores a lo largo de la titulación, no siempre atribuyen ese mérito a la formación recibida.

Palabras clave: representación social; escritura académica; didáctica de la escritura; formación universitaria.

\section{Abstract}

Learning what students think about academic writing becomes a valuable source of information that may significantly contribute to didactic proposals more suitable to educational needs. This study aims to inquire on notion about academic writing among students; whether they are being trained on this subject, and which training they are getting; and how they perceive themselves as writers since their beginning university studies until now. The target population was made up of 96 students in the Modern Languages program at the University of Deusto. They produced some narratives for the purposes of this cross-sectional qualitative study about students' social representations. Findings suggest that gaining access to a whole new discursive community poses new challenges for students, face to which academic training is insufficient and inadequate. Moreover, while they may perceive some improvement in their writing performance throughout their career, they do not always attribute it to the education received.

Keywords: social representation; academic writing; didactics of writing; higher education.

Received: 2017-11-23 / Accepted: 2018-10-18 / Published: 2019-01-24

DOI: 10.17533/udea.ikala.v24n01a05 
Doctoral inscrita en la Universidad de Deusto. Fecha de inicio y fecha final: septiembre del 2014 a octubre del 2018.

\section{RÉSUMÉ}

Les représentations que les étudiants ont sur l'écriture académique sont un savoir nécessaire à prendre en compte pour envisager des actions didactiques plus adéquates à leurs besoins de formation. Les objectifs de cette étude portent sur le type de concept qu'ont les étudiants sur l'écriture académique: s'ils considèrent avoir reçu une formation, quelle type de formation et comment, dès qu'ils commencent leurs études universitaires, ils se perçoivent quand ils écrivent. 96 étudiants en Licence en Langues Modernes de 1'Université de Deusto constituent le groupe d'étude. Ils ont produit des narrations qui sont objet de l'étude transversale qualitative sur leurs représentations sociales. Les résultats suggèrent que l'immersion dans une nouvelle communauté discursive soumet à de nouveaux défis ceux qui reçoivent une formation qu'ils considèrent insuffisante et peu adaptée, et, bien qu' ils perçoivent une amélioration dans leur trajet scriptural, ils ne l'attribuent pas toujours aux bienfaits de la formation reçue.

Mots clés : représentation sociale ; écriture académique ; compétence communicative ; réussite académique; formation universitaire. 


\section{Introducción}

La preocupación por las dificultades que experimentan los estudiantes universitarios en la redacción de textos académicos es una línea de investigación en auge en las últimas décadas. Desde estudios que se centran en el texto como un proceso (Carlino, 2004; Ceballos, 2014; Martínez, 2011) o como un producto (Álvarez y Boillos, 2015a; Guimarães, 2014; Tapia, Burdiles y Arancibia, 2003), hasta aquellos que se remontan a la propia formación en el aula (Bono y De la Barrera, 1998; Carlino, 2005, 2013) para producir textos académicos, el interés por esta cuestión afecta a todas las universidades (Ezeiza, 2012). No obstante, son pocos los estudios recientes, especialmente en el contexto español (Álvarez y Yániz, 2015; Cartolari y Molina, 2015; Castelló y Mateos, 2015; Castells, Mateos, Martín, Solé y Miras, 2015), que indagan sobre las representaciones sociales que los estudiantes construyen acerca de la escritura académica, su formación y los logros que alcanzan con ella.

El estudio que aquí se presenta se plantea dentro de una tesis doctoral que investiga la trayectoria en escritura académica de los estudiantes del grado en Lenguas Modernas, en la Universidad de Deusto, a través de la producción de una reseña. En esta tesis se concibe el texto no solo como una práctica académica, sino también como una práctica social, por lo que las representaciones de los estudiantes sobre la escritura académica se pueden cuestionar y modificar mediante diferentes intervenciones (Ortiz, 2015).

En este marco, esta investigación se propone conocer las representaciones sociales de los estudiantes universitarios de grado ${ }^{1}$ acerca de la escritura académica. Con ese objetivo general, la investigación se articula en torno a los siguientes objetivos específicos:

1 Se entiende por grado todos aquellos estudios universitarios de primer ciclo que siguen al bachillerato y dan acceso a los estudios de posgrado.
1. Indagar sobre la manera en que los estudiantes comprenden la actividad escritora.

2. Analizar si reciben formación sobre escritura académica y cómo la valoran.

3. Caracterizar la formación que desearían recibir.

4. Conocer la percepción que tienen sobre sí mismos como escritores de textos académicos desde que ingresaron en la universidad.

De este modo, los resultados aquí presentados inciden en los aspectos afectivos en relación con el texto académico, en las representaciones sociales de los estudiantes, mientras que, en otros ciclos de la investigación, mediante la teorización del análisis del discurso, se caracterizan sus producciones para conocer con qué nivel de eficacia dan respuesta a una situación comunicativa.

\section{Marco teórico}

\section{Escritura académica en la universidad}

La tarea de producir un texto académico es de gran complejidad, en varios sentidos: en primer lugar, exige que el autor del texto domine el código escrito del idioma que está empleando (Álvarez y Boillos, 2015b). En segundo lugar, su carácter histórico-social la sitúa como una tarea social que exige del autor conocer el contexto para poder contestar a los siguientes interrogantes: qué escribir, cómo escribirlo, para quién y con qué finalidad (Álvarez y Boillos, 2015b; Solé et al., 2005). En tercer lugar, se conforma como un proceso cognitivo orientado a un fin, que sigue un modelo de tres fases: planificación, textualización y revisión (Flower y Hayes, 1977, 1980). En cuarto y último lugar, es una herramienta de gran potencial epistémico, que ayuda a aprender y reorganizar el conocimiento (Álvarez y Boillos, 2015b; Bazerman et al., 2005; Carlino, 2005; Castelló, 2014; Castelló y Mateos, 2015).

Sin embargo, tradicionalmente, la escritura y los conocimientos disciplinares se han tratado por 
separado; es decir, se leía y se escribía en cada materia, pero el foco estaba fijado en los contenidos, no en enseñar a escribir sobre ellos. De esta manera, se interpretaba la escritura como un mero vehículo de comunicación del conocimiento dentro de las disciplinas (Bazerman, et al., 2005; Castelló, 2014).

Desde principios del siglo $\mathrm{xx}$, el interés por la enseñanza de la escritura académica aumentó de manera exponencial (Castelló, 2014), pero no se planteó, hasta finales de ese siglo, la idea de que los estudiantes se enfrentan a nuevas formas de leer y de escribir en la universidad, totalmente diferentes a lo que habían experimentado en su formación previa (Cadena, Narváez y Chacón, 2007).

Este nuevo enfoque para abordar la escritura académica dio lugar a toda una línea de investigación centrada en los géneros académicos (Henríquez y Canelo, 2014; Navarro, 2014; Parodi, 2009; Russell, 2002). Dentro de esta, los estudios que se preocupan por el proceso de apropiación de los géneros académicos por parte de los estudiantes distinguen cuatro esferas o ámbitos de uso, según los objetivos y las circunstancias de producción del texto: profesional (objetivo: difundir el conocimiento), académico (objetivo: obtener el grado de doctor), de enseñanza y aprendizaje (objetivo: aprendizaje de los conocimientos y formas disciplinares) y social (objetivo: establecer relaciones de carácter académico o afectivo). Entre estos, el estudiante sería un posible lector del ámbito profesional, pero participaría como escritor en los otros tres sistemas (Camps y Castelló, 2013).

Además de estos sistemas que ya, de por sí, delimitan las características de los géneros según el autor, el lector y el contexto de producción del texto, también habría que referirse a los estudios que se han fijado en la variedad disciplinar y en el efecto que tiene en los textos, ya que "entender las disciplinas implica entender sus discursos" (Hyland, 2007, p. 2; traducción de las autoras). Esto es, supone formar parte de una cultura disciplinar en la que hay unas normas, unos modos de escribir e investigar, unos grupos o tribus, y un conocimiento compartido. Cada cultura disciplinar se diferencia entre sí por sus dimensiones sociales y cognitivas, de tal manera que sus conductas, objetivos, intereses, relaciones y modalidades de comunicarse son diferentes (Becher y Trowler, 2001; Hyland, 2012; Navarro, 2014).

Por consiguiente, con estar "alfabetizado", entendido como ser capaz de leer y escribir textos, no es suficiente para que los estudiantes produzcan eficazmente los textos académicos que se les solicitan en las universidades (Carlino, 2005; 2013). La investigación en este respecto ha señalado, de manera reiterada, que las universidades tienen que enseñar explícitamente a los estudiantes a producir textos académicos que cumplan con las condiciones del estilo académico, porque el conocimiento adopta diferentes formas según la disciplina y el sistema o esfera de uso (Álvarez y Boillos, 2015b; Bazerman, et al., 2005; Castelló, 2014; Hyland, 2007).

\section{Representaciones sociales}

El estudio de las representaciones sociales en las ciencias sociales no tiene una larga trayectoria; de hecho, las primeras investigaciones se localizan en la década de los sesenta del siglo pasado (Cuevas y Mireles, 2016; Hebe, 2005).

Las representaciones sociales se refieren a las percepciones, lecturas o guías de acción de los individuos para interpretar un fenómeno, un objeto, una situación, etc. Dichas representaciones, además, están construidas por las experiencias y el bagaje de los diferentes grupos sociales (Jodelet, 2011).

A través de las representaciones sociales, se pueden llegar a entender prácticas sociales, cómo actúan los individuos y qué sentido tienen sus actitudes en un momento y lugar determinados (Ortiz, 2015). Permiten que esos individuos o grupos, con unas características comunes, destaquen lo que ellos juzgan relevante acerca de situaciones materiales (p. ej. lugares concretos), acontecimientos (por ejemplo, leyes, manifestaciones, guerras, etc.) o roles concretos 
(por ejemplo, docentes, estudiantes, familias, etc.) (Piña y Mireles, 2008).

No hay un claro acuerdo entre los autores que han intentado definir las representaciones sociales, pero es el enfoque procesual de Jodelet (2011) el que más se ajusta a las características de este estudio. Jodelet centra el foco de atención en cómo se sienten las personas respecto a una situación concreta, cómo ha sido su vivencia, cómo construyen la realidad y cuáles son las relaciones que se establecen. Distingue dos ámbitos: el de las rutinas (predecible) y el de los problemas (requiere una resolución). Ambos pueden ser estudiados según los intereses de la investigación (Seidmann, Bail, Azzollini, Thomé y Di lorio, 2006). Del estudio acerca de la vivencia de unos individuos o grupo social, se pueden extraer las representaciones sociales, desde los cuales se observan actitudes positivas o negativas hacia un fenómeno (Ortiz, 2015). En educación, este enfoque permite escoger una experiencia de enseñanza-aprendizaje concreta, para analizarla con el propósito de mejorar las intervenciones docentes.

Como ya se indicó antes, en el contexto español, este tipo de estudios acerca de las representaciones sociales de los estudiantes universitarios sobre la escritura académica son recientes y siguen siendo todavía más escasos que en el extranjero (Can y Walker, 2011; Christison y Krahnke, 1986; Hermerschmidt, 1999; Lea y Street, 1998; Lemke, 1990; Ortiz, 2015; Soler, 2013), pese a que autoras como Álvarez, Villardón y Yániz (2005) subrayan la necesidad de conocer la percepción de los estudiantes sobre la escritura y de sí mismos como escritores, como una fuente de información muy significativa acerca del valor que le dan a la escritura académica, cómo la definen, qué operaciones llevan a cabo cuando escriben, cómo se caracterizan como autores, etc. Desde ese conocimiento de la red de significados sobre la escritura académica que tejen los estudiantes, se pueden crear unidades didácticas que favorezcan de manera más eficaz el desarrollo de la composición escrita y su poder epistémico.

\section{Metodología}

Este es un estudio descriptivo, transversal, de corte cualitativo (con análisis cuantitativos puntuales para cálculo de medias), por ser esta la metodología de análisis que mejor se ajusta para dar respuesta a los objetivos de la investigación.

\section{Informantes y corpus}

El corpus de este estudio está conformado por las narrativas producidas individualmente por los 96 estudiantes universitarios del grado en Lenguas Modernas (LM) de la Universidad de Deusto (Bilbao).

En el estudio participaron la totalidad de los estudiantes de los cuatro cursos del grado en LM, que cumplen dos condiciones indispensables: no haber iniciado o terminado otro grado en la universidad previamente, y no haber repetido curso. Así, se constituyó un corpus de estudiantes de nueva matrícula en edades comprendidas entre los 17 y los 23 años.

Las narrativas se escribieron, además, en el segundo semestre del curso 2015/2016, para que los estudiantes de primero contaran con cierta experiencia como estudiantes universitarios.

\section{Instrumento de recogida de la información}

Para efectos de este estudio se creó una narrativa de tópicos que recolectara la experiencia de los estudiantes en lo referente a la escritura académica a lo largo del grado. Antes de hacer la recogida del corpus, se llevó a cabo un estudio piloto con estudiantes de Educación Primaria del área de didáctica de la lengua (por ser los más afines con los estudiantes de LM) de la misma universidad, quienes produjeron una narrativa sobre su propia experiencia en el grado al aprender a escribir textos académicos. Este piloto permitió realizar las modificaciones necesarias en la instrucción de la narrativa ${ }^{2}$ para orientar a los estudiantes en su redacción, de manera que

2 En el "Anexo" se aporta la instrucción de la narrativa. 
respondieran necesariamente a todos los objetivos de la investigación.

Tras modificar la instrucción de la narrativa a partir del análisis de los textos del estudio piloto, se recogió el corpus. Esto se hizo en las aulas, en horario de clase y en asignaturas en las cuales los profesores cedieron un espacio para esta labor, con el fin de asegurar la participación más alta posible.

\section{Procedimiento de análisis}

Para el análisis de la muestra se llevó a cabo una revisión cualitativa del contenido, centrada en los objetivos de la investigación. Este análisis fue realizado por las investigadoras de este estudio en paralelo, quienes pusieron en común los resultados obtenidos en cada una de sus fases para afinar y ajustar dicho instrumento, por lo que fue un proceso en constante retroalimentación.

Las fases fueron las siguientes (Cáceres, 2003):

1. Preanálisis. En la primera lectura del corpus se propusieron unos ítems (Ejemplos de ítems iniciales: "Importancia y necesidad de formación"; "Escribir como respuesta a lo que el profesor busca"; "Formación en estructurar textos académicos") que surgieron de la lectura del corpus. Esos ítems se refinaron en una segunda lectura de puesta en común.

2. Definición de las unidades de análisis. Después, se rediseñaron y agruparon las unidades de análisis y se hizo otra puesta en común para acordar su designación (Ejemplo de unidad de análisis: "Necesidad de formación"; "Escritura para ser evaluada"; "Formación sobre estructura”).

3. Elaboración del libro de códigos. A continuación se creó el "Libro de códigos", con las normas de inclusión y exclusión de cada unidad de análisis, para volver a clasificar todo el material (Ejemplo de criterio de inclusión: "Formación sobre estructura: alude específicamente a formación o indicaciones del profesor sobre cómo debe estructurarse un texto académico").

Una vez que el corpus se segmentó siguiendo las normas de análisis, se codificaron las unidades, para agilizar el estudio de los diferentes segmentos (Ejemplo de código: "Formación sobre estructura: FOREST") y se ha añadido esta información al "Libro de códigos".

4. Definición de las categorias. Esta categorización se hizo mediante la agrupación, bajo un mismo nombre, de los segmentos que tuviesen elementos comunes (Ejemplos de categorías: "Concepto de escritura académica"; "Formación en escritura académica”; "Metodologías de enseñanza percibidas"; "Metodologías de enseñanza deseadas"; "Valoración de la formación”, etc.).

\section{Resultados}

El análisis del corpus ha permitido identificar un total de tres dimensiones: concepto, formación y progreso. A continuación se presentan estas tres dimensiones, que permiten conocer diferentes aspectos de la percepción de los estudiantes sobre la escritura académica.

\section{Concepto de escritura académica}

Los estudiantes establecen una diferencia entre la manera de escribir en la universidad y lo que conocían de la formación previa. De hecho, el $25 \%$ de los estudiantes identifica y señala explícitamente que la forma de escribir en la universidad difiere de otras maneras que han conocido previamente (aludiendo a un estilo diferente a lo anterior).

Aunque estos datos ya son un indicativo de lo que los estudiantes piensan sobre la escritura académica, era interesante conocer qué es lo que opinaban por cursos y si esa percepción cambiaba. Por ese motivo, la figura 1 sitúa el porcentaje de estudiantes mencionado por curso que señaló una 
diferenciación entre el estilo de escritura en la universidad y otros estilos.

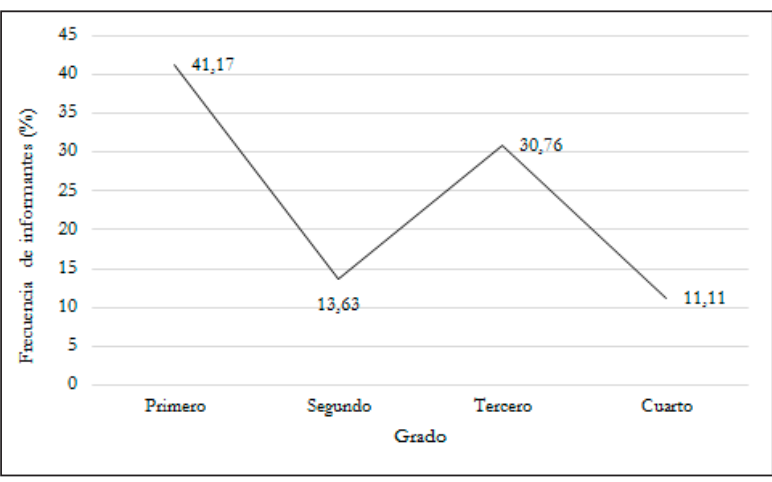

Figura 1. Frecuencia de informantes por curso que diferencian el estilo de la escritura de la universidad de otros.

Los porcentajes que se observan en la figura 1 indican que los estudiantes de los dos primeros cursos, y quizás también los de tercero, perciben de manera más destacada un cambio de estilo entre los estudios previos y la universidad. Estas divergencias entre cursos podrían estar relacionadas con el reciente ingreso en la universidad, especialmente en los dos primeros cursos; sin embargo, en el caso de tercero, la causa podría orientarse más hacia el efecto que pudiera tener la especialización del grado, en ese curso, en los estudiantes y en las tareas que deben afrontar en adelante. La inserción en una cultura disciplinar concreta resulta más evidente a medida que se centra el foco de atención de las programaciones en áreas más concretas.

Esa percepción de cambio en los estilos se extrae de expresiones como las siguientes: ${ }^{3}$

(1) Cuando comencé la universidad tuve algunos problemas a la hora de escribir los textos, ya que el estilo con el que hay que escribir nunca me lo habian exigido anteriormente (Informante 1.1). ${ }^{4}$

3 Las expresiones aquí recogidas como ejemplos se extraen de los testimonios escritos de los estudiantes. En estos, se resaltan datos que se interpretan en el análisis. Al final de cada ejemplo se señala, entre paréntesis, el informante al que corresponde el testimonio, mediante su código.

4 Los resaltados en los testimonios son de las autoras.
(2) En mi opinión, sí hay una gran diferencia en la manera de crear textos con respecto a bachiller (Informante 2.46).

(3) En primer y segundo año de carrera se dio un cambio notable en el enfoque que los textos tenian que tener, comparado al de bachiller; es por eso que el cambio se da desde primero (Informante 3.64).

Además de estas diferencias en el estilo, los informantes especificaron toda una serie de características concretas que permitieron identificar, de manera cualitativa, cómo conceptualizan la escritura académica. Los estudiantes de los dos primeros cursos se refirieron, sobre todo, a las peculiaridades de los textos, poniendo en relieve que son unos textos de mayor longitud; el tiempo y esfuerzo superior que requieren; la calidad del texto; el léxico especializado que se utiliza, y el registro (formal o informal). Hablan de "géneros", pero no advierten siempre la diversidad de la escritura como una práctica social. Incluso, a veces, la asocian con una actividad entendida y caracterizada según las exigencias de cada docente.

(4) Las diferencias que he notado al escribir textos académicos en la universidad es que son más largos que los que tenía que hacer en el instituto ${ }^{5}$ (Informante 1.30).

(5) Con la entrega de los primeros trabajos, uno se da cuenta del esfuerzo que estos requieren, y que no es algo que se pueda hacer en media hora (Informante 1.18).

(6) Otra gran diferencia ha sido el vocabulario empleado, ya que aquí el grado del vocabulario es superior y hay que saberlo utilizar adecuadamente (Informante 1.30).

(7) En la carrera de Lenguas Modernas he tenido que ir haciendo muchos trabajos, y bastantes de ellos, por no decir la gran mayoría, seguian las mismas pautas y tenian los mismos requisitos (Informante 1.25).

Luego, a medida que transcurren los cursos, estas indicaciones respecto a lo escrito se van diluyendo,

5 El instituto es un centro educativo de enseñanzas medias en el que se imparten cursos de Educación Secundaria Obligatoria y Bachillerato. 
hasta que, en los dos últimos cursos, cobran más importancia las peculiaridades relacionadas con la actividad escritora. En este caso, los estudiantes perciben la escritura como un proceso, como una tarea compleja y como una escritura que requiere mayor reflexión.

(8) Ahora me detengo a realizar un esquema previo antes de comenzar a redactar [...] y también he aprendido a realizar una revisión del trabajo final, con el objetivo de realizar correcciones si resultan necesarias (Informante 3.62).

Finalmente, estas caracterizaciones les hacen percibir la necesidad y, al mismo tiempo, la importancia de aprender a escribir textos académicos. Asimismo, establecen una relación entre el aprendizaje de ese tipo de textos con la inserción laboral.

(9) Antes de entrar a la universidad, solo había realizado uno o dos textos a este nivel y nunca me habia imaginado la importancia que tienen (Informante 1.16).

(10) La escritura académica es algo que, de mayor, teniendo en cuenta que quiero ser profesora, me va a venir muy bien (Informante 1.1).

(11) Pero en mi opinión, es una actividad a la que se deberia haber dado mayor relevancia, y para ello haber puesto a disposición de los estudiantes más asignaturas cuyo objetivo fuera la mejora y el perfeccionamiento de este apartado lingüístico (Informante 1.66).

Por lo tanto, en los cursos iniciales, los estudiantes tienen una percepción de la escritura como código vinculado a una serie de normas o convencionalismos formales. Más adelante, la experiencia y el bagaje en el grado los lleva a modificar esas percepciones hacia una concepción de la escritura como un proceso reflexivo y una tarea compleja. Al mismo tiempo que cambia su percepción de la escritura, también empiezan a comprender lo importante que es aprender a escribir textos académicos y surgen otro tipo de representaciones que asocian la escritura académica con la evaluación o con los textos profesionales. Sin embargo, no se observan representaciones sobre el dinamismo, la diversidad y el carácter dialógico de los textos académicos, en términos de Bajtín (1982).

\section{Formación en escritura académica}

En cuanto a la formación recibida en escritura académica, los estudiantes aportaron información desde la valoración de su idoneidad en términos generales, hasta la clasificación de la misma en iniciativas o metodologías empleadas en el aula, y las deseadas por ellos.

La figura 2 presenta el porcentaje de informantes que demuestra insatisfacción con la formación recibida en escritura académica.

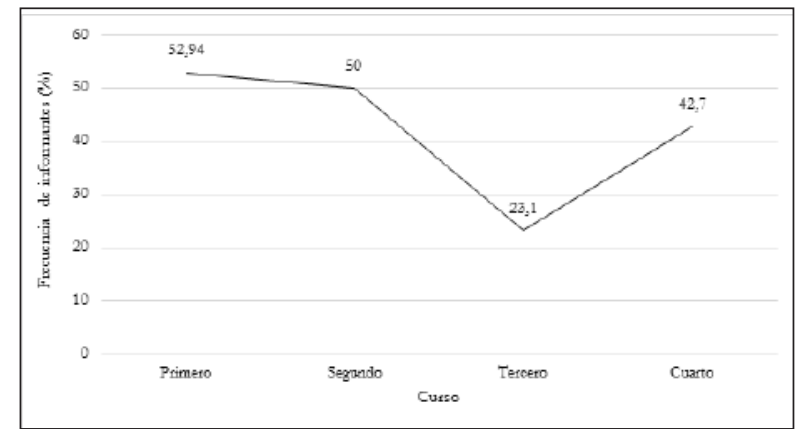

Figura 2. Frecuencia de informantes por curso que valoran su formación en escritura académica como insuficiente.

Como se observa en la figura 2, hay un alto porcentaje de estudiantes por curso que califica de insuficiente la formación en escritura académica. Este porcentaje se divide de manera desigual entre los cuatro cursos. En los dos primeros años, durante el período de transición de la educación media a la superior, se observa que un mayor número de estudiantes realiza una valoración de este tipo, mientras que, en tercero y en cuarto, ese porcentaje baja al menos un $20 \%$. Ahora bien, habría que señalar, en cuarto, en referencia a ese pico del $42,7 \%$ respecto al curso anterior, que los estudiantes se enfrentan a la producción de un texto académico de investigación, como es el trabajo de fin de grado, que implica un desafío retórico-discursivo mayor que otros textos que hayan producido previamente. 
En este sentido, sobre la insuficiencia de la formación, los estudiantes se expresaron de la siguiente manera:

(12) La verdad es que solo hemos escrito un ensayo en lo que llevamos de curso; nadie nos ha enseñado realmente a hacerlo mejor de lo que lo haciamos en el bachillerato (Informante 1.4).

(13) Creo firmemente que tampoco se han esforzado mucho en que aprendamos a escribir textos $\mathrm{o}$, al menos, ese no ha sido el objetivo principal del profesor (Informante 2.38).

(14) A excepción de una asignatura en segundo curso, en la que se realiza una monografía, considero que apenas enseñan a redactar cualquier tipo de texto (Informante 4.93).

Como bien se ha comentado hasta aquí, los informantes demuestran su insatisfacción con la formación recibida, pero eso no significa que no percibieran que se hubieran llevado a cabo iniciativas para el aprendizaje. De hecho, los informantes distinguen entre metodologías para el aprendizaje, iniciativas concretas y características de esa formación.

Entre las metodologias de aprendizaje, lo que más destacan los estudiantes (el 43,7 \%) es la realización de prácticas discursivas diferentes. Entre estas, refieren desde textos académicos tan concretos como el "ensayo", el "abstract" o la "monografía", hasta textos de una definición más vaga por parte del docente, como "redacciones", "trabajos escritos" o "proyectos".

Asociadas con estas iniciativas para el aprendizaje de la escritura académica, a continuación se refieren a las pautas $(28,1 \%)$ que da el docente para escribir el texto que solicita como tarea. Estas suelen estar orientadas hacia los aspectos más formales del texto: longitud del texto, portada, índice, tamaño de letra, etc., sin llegar a abarcar otro tipo de cuestiones relacionadas con la estructura, los objetivos comunicativos o la audiencia del texto. Algunos ejemplos de estos:

(15) En las siete asignaturas que he cursado hasta el momento, he tenido que escribir todo tipo de textos: resúmenes, ensayos, artículos, reseñas... (Informante 1.6).

(16) La mayoría de las veces se nos dice que tenemos que hacer un trabajo, nos dan el tema y nosotros somos los que tenemos que desarrollarlo, sacando la estructura, etc. Por ejemplo, en una asignatura nos han dado un tema y la extensión que el trabajo tiene que tener, y nada más (Informante 2.40).

Con menor frecuencia que las metodologías anteriores, algunos informantes destacan que algunos profesores $(18,7 \%)$, que son conscientes de las dificultades que experimentan los estudiantes para producir el texto, demuestran una mayor implicación, al orientar de forma más explícita la tarea escritora.

(17) Sin embargo, también hay profesores que se implican y ayudan a los estudiantes para mejorar. Y esto es algo que deberían hacer todos (Informante 1.1).

Asimismo, perciben que la metodología de enseñanza cambia de un curso a otro (13,5\%), graduándose para alcanzar un mayor nivel de complejidad según avanzan en la carrera. Con ello, cada vez depende más del estudiante aprender a producir los textos que se le solicitan (13,5\%), esto es, no asocian la mayor dificultad con la aparición de nuevos textoso con un mayor nivel de exigencia en la elaboración de los textos respecto a los que producían previamente, sino con la actitud del docente, que presupone un nivel de dominio del lenguaje académico que aún no han alcanzado. Ejemplos de esto son:

(18) El mayor impacto que sufrí fue el cambio de primero a segundo. En primero se abordó un poco el tema de la bibliografía, pero sin darle gran importancia. Sin embargo, la llegada a segundo resultó más complicada de lo que parecía; se nos exigía saber citar, usar una buena estructura del texto y usar un lenguaje culto y formal (Informante 2.56).

(19) La experiencia de aprender a escribir textos académicos ha sido mala. La mayoría de los profesores dan por hecho que ya sabemos hacer muchas cosas y no dan explicaciones (Informante 3.62). 
Por último, unos pocos mencionan la entrega de materiales $(4,1 \%)$ para aprender a escribir, sin ninguna explicación del docente:

(19) Para aprender a escribir un texto, los profesores suben PDFs al ALUD ${ }^{6}$ para explicarnos cómo hay que hacerlos. Sin embargo, simplemente con eso no vale (Informante 1.27).

Por otro lado, como iniciativas concretas para el aprendizaje de la escritura académica, a partir de segundo se empieza a señalar la existencia de unas asignaturas (22,9\%), todas ellas del área de lingüística, que se dedican exclusivamente a la enseñanza de los textos académicos.

(20) A lo largo de la carrera hemos tenido varias asignaturas, con el fin de mejorar nuestra forma de escribir textos académicos (Informante 3.57).

(21) A lo largo de mi carrera hemos tenido una serie de asignaturas, tanto en inglés como en español, que trataban sobre el uso de lengua en el ámbito académico. Nos trataban de enseñar los conceptos básicos de la escritura académica y promover actividades que nos ayudasen a adquirir las competencias necesarias en el ámbito académico (Informante 4.71).

Por último, de manera más superficial, los informantes se refieren a las características de la formación que perciben, como el hecho de que se le esté dando mayor importancia a la escritura en otras lenguas (6,2 \%), mientras que la exigencia en la evaluación es mayor en castellano (2,1\%). Asimismo, perciben mayor formación en la comunicación oral $(1 \%)$, cuando es el texto escrito el que se valora con más dureza $(1 \%)$.

(22) Entiendo que sea importante el aprendizaje de la escritura académica en una segunda lengua. Sin embargo, ya que a la hora de redactar en lengua española, el nivel de exigencia es mayor, y personalmente pongo en duda mi capacidad de llegar a esos requisitos (Informante 1.10).

(23) El profesorado se ha centrado más en explicarnos cómo hacer una exposición oral y no tanto en

6 Plataforma digital de aprendizaje de la Universidad de Deusto. cómo deberíamos realizar las actividades por escrito (Informante 1.9).

(24) Opino que la expresión escrita es tan importante como la oral, pero a la primera se le da más valor y se le exige más (Informante 1.10).

Junto a todo lo anterior, los informantes se refieren también a una serie de contenidos enseñados: el tratamiento de la información y la citación, la estructura del texto, la objetividad, el registro formal, los géneros y el léxico. De todos estos, destacan, además, aquellos contenidos en los que más se ha insistido, como la estructura del texto y el tratamiento de la información y la citación, pero como meros aspectos formales del texto, sin llegar a comprender el efecto que pueden tener en el mensaje y en su forma.

Finalmente, al tiempo que identifican estas metodologías, iniciativas o características y contenidos de la formación que están recibiendo, también expresan su deseo de que esa formación sea diferente en algunos aspectos. Solicitan, de manera muy destacada, la necesidad de una retroalimentación (feedback) más detallada, para la mejora de los textos, y que aquella sea individualizada.

Relacionado con este asunto, también comentan la posibilidad de poder escribir más de una vez los textos con la ayuda de las correcciones del docente, para poder mejorarlos antes de entregar el producto final.

Asimismo, asocian el aprendizaje de la escritura de textos con la lectura, una tarea que no creen realizar lo suficiente en la universidad, por lo que solicitan que se lean más textos académicos.

Por último y en número más reducido, unos pocos necesitan hacer más textos y que los formen en la estructura del texto y en un vocabulario más técnico.

En resumen, aquello que presentan los informantes con lo que se está realizando en las aulas (metodologías, iniciativas, características de la formación, etc.) se contrapone con el tipo de metodología que 
ellos desearían. Se oponen a la imagen del docente que da por supuestos ciertos conocimientos y solo explica cuando advierte un problema; que da materiales, sin explicarlos, como complemento para ayudar a escribir los textos que solicita, o que solo $\mathrm{da}$ indicaciones que se refieren a aspectos formales del texto. Por el contrario, los estudiantes exigen la figura de un docente implicado, que los acompañe durante el proceso de producción de los textos académicos que les solicita, para que puedan escribir textos académicos de mayor calidad, con progresiva autonomía.

\section{Percepción de sí mismos como escritores}

Como última dimensión de análisis, en cuanto a su progreso a lo largo del grado, la mayoría de los estudiantes $(73,9 \%)$ perciben una mejoría en su forma de escribir desde que ingresaron en la universidad. Es en cuarto donde esa percepción, aun siendo la predominante, desciende bastante en comparación con los cursos anteriores.

Asimismo, un bajo porcentaje de estudiantes $(13,5 \%)$, concentrados en los dos primeros cursos, no perciben ninguna mejoría por el momento, y otro resto de estudiantes en primero (5,8 \%) creen que incluso empeoraron en la escritura de los textos. Estos dos últimos casos pueden deberse a que aún no han tenido suficiente experiencia en el grado en la escritura de textos académicos.

Algunos ejemplos ilustrativos de estas diversas percepciones son:

(25) En mi experiencia en las diferentes asignaturas que hemos tenido y estamos teniendo actualmente en el grado, puedo afirmar que mi mejora a la hora de escribir los diferentes textos académicos o ensayos a [sic] sido enorme (Informante 1.21).

(26) En lo referente a mi manera de redactar desde que comenzamos este grado, creo que ha mejorado tanto en inglés como en castellano (Informante 3.65).

(27) Comencé la universidad hace casi seis meses y no he notado ninguna mejoria en lo que se refiere a mi modo de escribir. Diría que incluso en bachi- llerato escribia mejor, ya que se me explicaba cómo redactar cada tipo de texto y lo hacía con más frecuencia (Informante 1.6).

Dentro de esa mejoría, además, los informantes señalaron una serie de aspectos específicos en los que mejoraron en la universidad: estructurar textos, una escritura más reflexiva y el uso de un léxico más variado. De modo más anecdótico, otros se refirieron a los aspectos formales del texto, al enunciador, el tratamiento de la información y la citación, las normas académicas, el proceso de redacción del texto, el uso de conectores, el estilo, y otros aspectos de carácter más general, como la mejoría en idiomas o en castellano.

(28) En cambio ahora a través [sic] de las pautas que me han otorgado, puedo decir que he mejorado en esa faceta y que ahora se [sic] escribir mejor, con un léxico mas [sic] variado y consciente de porqué [sic] escribo una cosa y de por qué la utilizo de una manera $o$ [sic] otra (Informante 1.8).

(29) He aprendido a estructurar un texto (o al menos eso creo) (Informante 2.43).

(30) [...] y puedo hilar mejor las ideas (más en inglés que en castellano), aunque supongo que me falta mejorarlo (Informante 2.47).

No son esos aprendizajes lo único que habría que señalar acerca de esa mejoría. También muestran cómo se llevó a cabo el proceso para adquirir esos aprendizajes. En este caso, más que asociarlo a una formación concreta en el grado, se refieren a un proceso de aprendizaje por evolución personal, mediante la práctica con la retroalimentación recibida en una tarea, para mejorar para la próxima tarea o para otras materias; y por la práctica de escribir y escribir. Asimismo, los estudiantes de cuarto se refieren a las estancias en el extranjero en el curso anterior, donde han percibido que se les enseñaba, de manera más explícita, a escribir un texto académico en el aula.

\section{Discusión}

En relación con el primer objetivo de la investigación, esto es, qué entienden los estudiantes 
por escritura académica, ya desde el primer curso se advierte lo mismo que señala Carlino (2008, p. 164) en un estudio sobre el espacio que ocupan la lectura y la escritura en diferentes universidades, acerca de que la escritura no es "una habilidad básica, general y, por tanto, transferible” (Carlino, 2008, p. 164). Como también muestra Hyland (2012), todo parece indicar que la exposición a tener que enfrentarse al reto de escribir textos académicos desde que ingresan en la universidad, conduce a los estudiantes hacia una percepción de la escritura más como práctica social y como propia de la nueva comunidad discursiva en la que ingresan. No obstante, hay estudiantes que perciben la escritura como un instrumento de evaluación, que cambia en consonancia con las preferencias de cada docente. Esta idea se confrontaría, sin embargo, con lo que destacan algunos estudiantes: la concepción de la escritura como una herramienta útil para el ejercicio profesional.

Es verdad que algunos estudiantes identifican rasgos específicos de la escritura académica asociados tanto a lo escrito como a la actividad escritora, que se relacionan con concepciones que vinculan la escritura como una práctica social. Ahora bien, estas evidencias no con comunes a todos los estudiantes, manifestándose divergencias, como el conflicto entre género, diversidad y ámbito. Hablan de "géneros", pero también, en ocasiones, identifican unas características de la escritura académica como universales y abstractas, sin ser conscientes de su vinculación con el contexto, el propósito comunicativo o el destinatario (Carlino, 2003; Casamiglia y Tusón, 2001; Gutiérrez-Rodríguez y Flórez, 2011).

En resumen, la percepción que manifiestan los estudiantes se aleja de manera clara de asumir la escritura como una habilidad básica o una labor instantánea que no requiere de una planificación detenida, y parece orientarse a considerarla como una práctica social y como un proceso cognitivo que exige mayor reflexión y esfuerzo. Sin embargo, también es cierto que se manifiestan otro tipo de creencias, comportamientos y prácticas vinculadas a otro tipo de representaciones más tradicionales, acerca de la escritura académica como un medio de evaluación de los conocimientos de los estudiantes.

Respecto al segundo objetivo de esta investigación, referido a la formación recibida para aprender a escribir textos académicos, se manifiesta un clima general de insatisfacción, que se presenta con más fuerza en los primeros cursos, lo cual podría estar vinculado al salto que perciben entre la educación media y la educación superior en relación con la escritura. Nuevamente, esa insatisfacción se intensifica en el último curso, quizás determinada por los requerimientos exigidos para la elaboración de un texto de la complejidad del trabajo de fin de grado en ese período formativo.

Por otra parte, en relación con las metodologías de enseñanza de la escritura que los informantes señalan, estas coinciden con lo que Carlino (2013) denomina "modelo de trabajo centrado en los extremos": se solicita el trabajo, se proporcionan técnicas y pautas, y se corrige el producto. Esta manera de enseñar a escribir condiciona tanto el proceso de la actividad como la retroalimentación recibida, quedando fuera de la actividad pedagógica lo que Carlino, Iglesia y Laxalt (2013) denominan el "trabajo durante el proceso de realización de la actividad escritora”. Es en esta línea en la que se sitúan los deseos de los estudiantes cuando manifiestan necesitar una retroalimentación más detenida e individualizada en el transcurso del proceso de elaboración de los textos.

Los resultados a los que se llega en este aspecto de la investigación coinciden con los obtenidos por Lea y Street (1998) en un estudio sobre las percepciones de los estudiantes y los profesores universitarios respecto a la composición del texto; asimismo, con los hallazgos de Álvarez y Yániz (2015) en una investigación donde analizaron prácticas escritoras en la universidad; y con los de Castelló y Mateos (2015) en un estudio sobre las representaciones sociales de estudiantes y profesores en la universidad española. En todos estos estudios, hay algún tipo de formación sobre escritura académica, pero es una formación más bien escasa sobre aspectos puntuales de la escritura, 
en diferentes momentos, en distintas materias y sin una coordinación entre docentes (se repiten contenidos), que no permite que el estudiante saque verdadero provecho para mejorar sus textos.

En lo que respecta a cómo se perciben a sí mismos como escritores, el tercer objetivo de este estudio, se constata la percepción de mejoría de manera más intensa en el tercer curso; en los otros cursos, por el contrario, no se manifiestan tantos testimonios positivos. Esto permite al estudio subrayar la relación entre la formación que reciben los estudiantes de cada curso (el segundo objetivo del estudio) con la percepción de sí mismos como escritores desde que ingresaron al grado (el tercer objetivo), ya que se constatan unos resultados relativos a la insatisfacción con la formación y su percepción como escritores, que parecen estar en paralelo. Por tanto, no se puede soslayar la influencia que en el nivel afectivo de los estudiantes presenta el estar inmersos en una comunidad discursiva nueva que les plantea nuevos retos, y las exigencias del trabajo de fin de grado.

En definitiva, de estas representaciones de los estudiantes de grado se extraen orientaciones importantes de cara a diseñar prácticas docentes conducentes a una mejora de la escritura académica. Entre estas, el conjunto de resultados relativos al concepto, la formación y el logro alcanzado en escritura académica permiten subrayar, al menos, dos aspectos clave para abordar los procesos de enseñanza-aprendizaje de la escritura académica con mayor eficacia:

1. La conveniencia de enfrentar a los estudiantes a llevar a cabo actividades escritoras que les planteen retos sobre el plan retórico en el que se ubica la actividad, pero también retos sobre las convenciones con las que se construye el conocimiento disciplinar, además de, por supuesto, cuestiones de tipo idiomático.

2. La importancia y la necesidad de abordar el desarrollo de la escritura académica mediante un plan de trabajo que no incida solo, utilizando los términos de Carlino, Iglesia y Laxalt (2013, p. 119), en el "trabajo en los extremos", sino también en el "trabajo durante" el proceso de elaboración de los textos.

Estos hallazgos, entonces, abren un camino interesante para el diseño de futuras programaciones que integren la formación en escritura académica. Ahora bien, todavía son unas conclusiones que deben ser tratadas con cautela, bien por tratarse de un estudio transversal (completado por diferentes estudiantes de diferentes cursos), o bien porque el propio instrumento de recogida de la información (la narrativa) puede que no haya permitido que los estudiantes profundicen lo suficiente en esta cuestión.

\section{Referencias}

Álvarez, M., Villardón, L., y Yániz, C. (2005). Estilos de aprendizaje y actitudes hacia la escritura en estudiantes universitarios. IIIJornadas de Redes de Investigación en Docencia Universitaria: la configuración del Espacio Europeo de Educación Superior. Universidad de Alicante, Alicante. Recuperado de https:// rua.ua.es/dspace/bitstream/10045/20044/4/COMUNICACIONES.pdf

Álvarez, M., y Boillos, M. (2015a). La competencia comunicación escrita. En L. Villardón-Gallego, Competencias genéricas en educación superior (pp. 5577). Madrid: Narcea, S. A.

Álvarez, M., y Boillos, M. (2015b). La producción escrita de los estudiantes universitarios de nuevo ingreso. Magis, Revista Internacional de Investigación en Educación, 8(16), 71-90. DOI: http://dx.doi. org/10.11144/Javeriana.m8-16.peeu

Álvarez, M., y Yániz, C. (2015). Las prácticas escritas en la universidad española. Cultura y Educación, 27(3), 594-628. Dor: http://dx.doi.org/10.1080/1 1356405.2015 .1072356 .

Bajtín, M. (1982). El problema de los géneros discursivos. En M. Bajtín, Estética de la creación verbal (pp. 248293). México: Siglo XXI.

Bazerman, C., Little, J., Bethel, L., Chavkin, T., Fouquette, D., y Garufis, J. (2005). Reference guide to writing across the curriculum. Indiana: Parlor Press y The WAC Clearinghouse.

Becher, T., y Trowler, P. (2001). Academic Tribes and Territories. Buckingham: SRHE y Open University Press.

Bono, A., y De la Barrera, S. (1998). Los estudiantes universitarios como productores de textos. Una ex- 
periencia de docencia compartida. Revista Lectura y Vida, año XIX(4), 13-20. Recuperado de http:// www.lecturayvida.fahce.unlp.edu.ar/numeros/ a19n4/19_04_Bono.pdf

Cáceres, P. (2003). Análisis cualitativo de contenido: una alternativa metodológica alcanzable. Psicoperspectivas, 2(1), 53-82. http://www.redalyc.org/articulo. oa? id=171018074008

Cadena, S., Narváez, E., y Chacón, M. (2007). Alfabetización académica: una de las responsabilidades de la educación superior. Encuentro Nacional de Discusión sobre Politicas Institucionales para el Desarrollo de la Lectura y la Escritura en la Educación Superior (pp. 1-19). Bogotá: Universidad Sergio Arboleda. Recuperado de https://media.utp.edu.co/referencias-bibliograficas/uploads/referencias/ponencia/ sonia-cadena-autonoma-de-occidentepdf-E6vZRarticulo.pdf

Camps, A., y Castelló, M. (2013). La escritura académica en la universidad. Revista de Docencia Universitaria (REDU), 11(1), 17-36. Recuperado de http://red-u. net/redu/files/journals/1/articles/579/public/5792168-1-PB.pdf

Can, G., y Walker, A. (2011). A model for doctoral students' perceptions and attitudes toward written feedback for academic writing. Research in Higher Education, 52(5), 508-536. DOI: https://doi.org/10.1007/ s11162-010-9204-1

Carlino, P. (2003). Alfabetización académica: un cambio necesario, algunas alternativas posibles. Educere, 6(20), 409-420. Recuperado de http://www.redalyc. org/articulo.oa? id=35662008

Carlino, P. (2004). El proceso de escritura académica: cuatro dificultades de la enseñanza universitaria. $E d u-$ cere, 8(26), 321-327. Recuperado de http://www. redalyc.org/articulo.oa? $\mathrm{id}=35602605$

Carlino, P. (2005). Escribir, leer y aprender en la universidad. Una introducción a la alfabetización académica. El Salvador: Fondo de Cultura Económica de Argentina, S. A.

Carlino, P. (2008). Leer y escribir en la universidad, una nueva cultura. ¿ Por qué es necesaria la alfabetización académica? En E. Narváez y S. Cadena, Los desafios de la lectura y la escritura en la educación superior: caminos posibles (pp. 154-194). Cali: Universidad Autónoma de Occidente.

Carlino, P. (2013). Alfabetización académica diez años después. Revista Mexicana de Investigación Educativa, 18(57), 355-381. Recuperado de http://www.redalyc.org/articulo.oa? $\mathrm{id}=14025774003$
Carlino, P., Iglesia, P., y Laxalt, I. (2013). Concepciones y prácticas declaradas de profesores terciarios en torno al leer y escribir en las asignaturas. Revista de Docencia Universitaria (REDU), 11(1), 105-135. DOI: https://doi.org/10.4995/redu.2013.5594

Cartolari, M., y Molina, M. (2015). Modos de leer y escribir en Historia: perspectivas de alumnos y profesores de la formación docente inicial. Revista de Docencia Universitaria (REDU), 13(3), 235-263. DOI: https:// doi.org/10.4995/redu.2015.5428

Casamiglia, H., y Tusón, A. (2001). Las cosas del decir. Manual de análisis del discurso. Barcelona: Ariel.

Castelló, M. (2014). Los retos actuales de la alfabetización académica: estado de la cuestión y últimas investigaciones. En I. Ballano e I. Muñoz, Escribir en el contexto académico (pp. 153-176). Bilbao: Universidad de Deusto.

Castelló, M., y Mateos, M. (2015). Las representaciones de profesores y estudiantes sobre la escritura académica en las universidades españolas. Cultura y Educación, 27(3), 477-503. DoI: http://dx.doi.org/10.1080/1 1356405.2015 .1072357

Castells, N., Mateos, M., Martín, E., Solé, I., y Miras, M. (2015). Perfiles de competencias y concepciones sobre la escritura académica en estudiantes universitarios. Cultura y Educación, 27(3), 569-593. DOI: https://doi.org/10.1080/11356405.2015.1072358

Ceballos, W. (2014). Escritura de textos académicos. Educación \& Pensamiento, 21, 84-93. Recuperado de http://revista.colegiohispano.edu.co/ojs/index. $\mathrm{php} /$ tomo21/article/view/47

Christison, M., y Krahnke, K. (1986). Student perceptions of academic language study. TESOL Quarterly, 20(1),6181. DoI: https://doi.org/10.2307/3586389

Cuevas, Y., y Mireles, O. (2016). Representaciones sociales en la investigación educativa. Estado de la cuestión: producción, referentes y metodología. Perfiles Educativos, XXXVIII(153), 65-83. Recuperado de http:// www.redalyc.org/articulo.oa?id=13246712005

Ezeiza, J. (2012). Bases para la evaluación del dominio de las formas disciplinares de comunicación y de los usos lingüísticos especializados en el Espacio Europeo de Educación Superior (EESs). Revista Nebrija de Lingüistica Aplicada, (12), 88-119. Recuperado de https:// www.nebrija.com/revista-linguistica/files/articulosPDF/articulo_5280b4c7c4020.pdf

Flower, L., y Hayes, J. (1977). Problem-solving strategies and the writing process. College English, 39(4), 449461. DoI: https://dx.doi.org/10.2307/375768 
Flower, L., y Hayes, J. (1980). The dynamics of composing: Making plans and juggling constraints. En L. G. Steinberg, Cognitive processes in writing (pp. 31-50). Erlbaum: New Jersey.

Guimarães, M. O. (2014). Pode ser... poderia ser... O uso de modalizações na escrita acadêmica. Revista Brasileira de Linguística Aplicada, 14(1), 175-197. DOI: https://dx.doi.org/10.1590/S1984-63982014 000100009

Gutiérrez-Rodríguez, J., y Flórez, R. (2011). Enseñar a escribir en la universidad: saberes y prácticas de docentes y estudiantes universitarios. Magis, 4(7), 137-168. Recuperado de http://revistas.javeriana.edu.co/index.php/MAGIS/article/view/3561

Hebe, L. (2005). Representaciones sociales: una manera de entender las ideas de nuestros alumnos. Revista Electrónica de la Red de Investigación Educativa, 1(3), 1-17. Recuperado de http://revista.iered.org/ v1n3/pdf/llacolla.pdf

Henríquez, R., y Canelo, V. (2014). Géneros históricos y construcción de la significación histórica: el caso de los estudiantes de Licenciatura en Historia. ONOMÁZEIN, (Número Especial IX ALSFAL), 138-160. Recuperado de http://onomazein.letras.uc.cl/Articulos/N_ALSFAL/ESP_8_Henriquez_FINAL.pdf

Hermerschmidt, M. (1999). Foregrounding background in academic learning. En C. Jones, J. Turner y B. Street, Students Writing in the University. Cultural and Epistemological Issues (pp. 5-16). Amsterdam: John Benjamins Publishing Company.

Hyland, K. (2007). Disciplinary Discourses: Social Interactions in Academic Writing. Michigan: Ann Arbor, University of Michigan Press.

Hyland, K. (2012). Disciplinary Identities. Individuality and Community in Academic Discourse. Cambridge: Cambridge University Press.

Jodelet, D. (2011). Aportes del enfoque de las representaciones sociales al campo de la educación. Espacios en Blanco, 21(1), 133-154. Recuperado de http://www.scielo.org.ar/scielo.php?script $=$ sci arttext\&pid $=$ S1515-94852011000100006\&lng $=\mathrm{e}$ s\&tlng=es

Lea, M., y Street, B. (1998). Student writing in higher education: An academic literacies approach. Studies in Higher Education, 23(2), 157-172. Recuperado de https://www.kent.ac.uk/teaching/documents/ qualifications/studwritinginhe.pdf

Lemke, J. (1990). Talking Science: Language, Learning, and Values. Norwood, NJ: Ablex.
Martínez, J. (2011). La planificación textual y el mejoramiento de la escritura. Infancias, 9(2), 35-47. Recuperado de https://dialnet.unirioja.es/servlet/ articulo ?codigo $=3653126$

Navarro, F. (2014). Manual de escritura para carreras de humanidades. Buenos Aires: Facultad de Filosofía y Letras de la Universidad de Buenos Aires.

Ortiz, E. (2015). La escritura académica en el contexto universitario (Pregrado). Zona Próxima, (22), 1-16. Recuperado de http://www.redalyc.org/articulo. oa?id=85339658002

Parodi, G. (2009). Written genres in university studies: Evidence from an academic corpus of spanish in four disciplines. En C. Bazerman, A. Bonini, y D. Figueiredo, Genre in a Changing World (pp. 483-501). Indiana: Parlor Press y The wAC Clearinghouse.

Piña, J., y Mireles, O. (2008). La perspectiva sociológica de las representaciones sociales para el estudio de la globalización y la universidad. Convergencia con Europa y cambio en la universidad: XI Conferencia de Sociología de la Educación. Santander, España. Recuperado de https://dialnet.unirioja.es/servlet/ articulo ?codigo $=2376684$

Russell, D. (2002). Writing in the Academic Discipline. A Curricular History. Estados Unidos: Southern Illinois University Press.

Seidmann, S., Bail, V., Azzollini, S., Thomé, S., y Di lorio, J. (2006). La representación social de la vida cotidiana en jóvenes: naturalización y cambio. Anuario de Investigaciones, XIV, 287-294. Recuperado de http://www.scielo.org.ar/scielo.php?script =sci_ arttext\&pid=S1851-16862009000100027\&lng=e $\mathrm{s} \& \mathrm{t} \operatorname{lng}=\mathrm{pt}$

Solé, I., Mateos, M., Miras, M., Martín, E., Castells, N., Cuevas, I., y Gràcia, M. (2005). Lectura, escritura y adquisición de conocimientos en Educación Secundaria y Educación Universitaria. Infancia y Aprendizaje, 28(3), 329-347. DoI: http://dx.doi. org/10.1174/0210370054740241

Soler, S. (2013). Representaciones sociales de la escritura académica en contextos de bilingüismo e interculturalidad. Signo y Pensamiento, XXXII(62), 64-80. Recuperado de http://revistas.javeriana.edu.co/index.php/signoypensamiento/article/view/5334

Tapia, M., Burdiles, G., y Arancibia, B. (2003). Aplicación de una pauta diseñada para evaluar informes académicos universitarios. Revista Signos, 36(54), 249-257. DoI: http:// dx.doi.org/10.4067/S0718-09342003005400009 


\section{Anexo. Narrativa}

Narra en un texto (de unas 150 palabras) tu experiencia en aprender a escribir los textos (informe, ensayo, monografía, trabajos, proyectos...) que te piden en el grado en Lenguas Modernas y cómo valoras esa experiencia. Para ello...

- Identifica las iniciativas a nivel de grado, asignaturas, temas o ejercicios que se han realizado en el grado para el aprendizaje de la escritura académica.

- Reflexiona sobre ti mismo, sobre cómo ha cambiado tu forma de escribir desde que comenzaste a estudiar en la universidad.

- Valora tu experiencia en el grado aprendiendo a escribir textos académicos.

- Adicionalmente, puedes mencionar también cualquier propuesta, opinión, vivencia, etc., relativa a tu experiencia aprendiendo a escribir textos en la universidad, que consideres que sea relevante.

How to reference this article: Romero-González, A.N., y Álvarez-Álvarez, M. (2019). Representaciones sociales de los estudiantes universitarios de grado sobre la escritura académica. Íkala, Revista de Lenguaje y Cultura, 24(1), 103-118. Dor: 10.17533/udea.ikala.v24n01a05 